
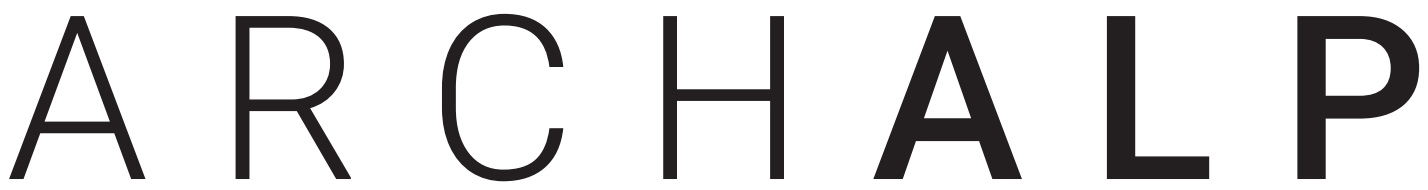

Rivista internazionale di architettura e paesaggio alpino / Revue internationale d'architecture et de paysage dans les Alpes / Internationale Zeitschrift für Alpine Architektur und Landschaft / Revija za alpsko arhitekturo in pokrajino / International journal of alpine architecture and landscape

Rer una nuota abitabilita delle Alpi.

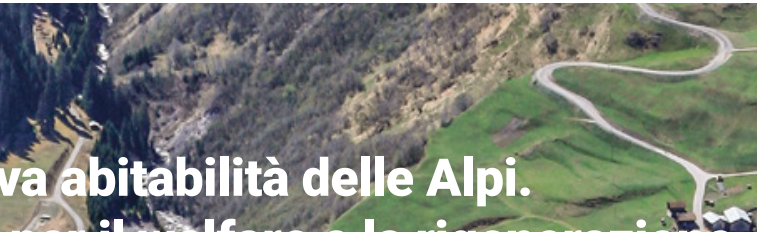
Architeture pet ilf lfare e la rigenerazione For a new inhabifability of the Alps. Architectures for welfare ând regeneration $/$ Pour une nouvelle habitabilité des Alpes. Architectures pour le welfare et la régéhérationy Für eine núue Bewohnbarkeit in den Alpen. Architekturen für Wohf ahrt und Regeneration / Za novo. bivalnost v Alpah, arhitekture za dobrobit in regeneracijo
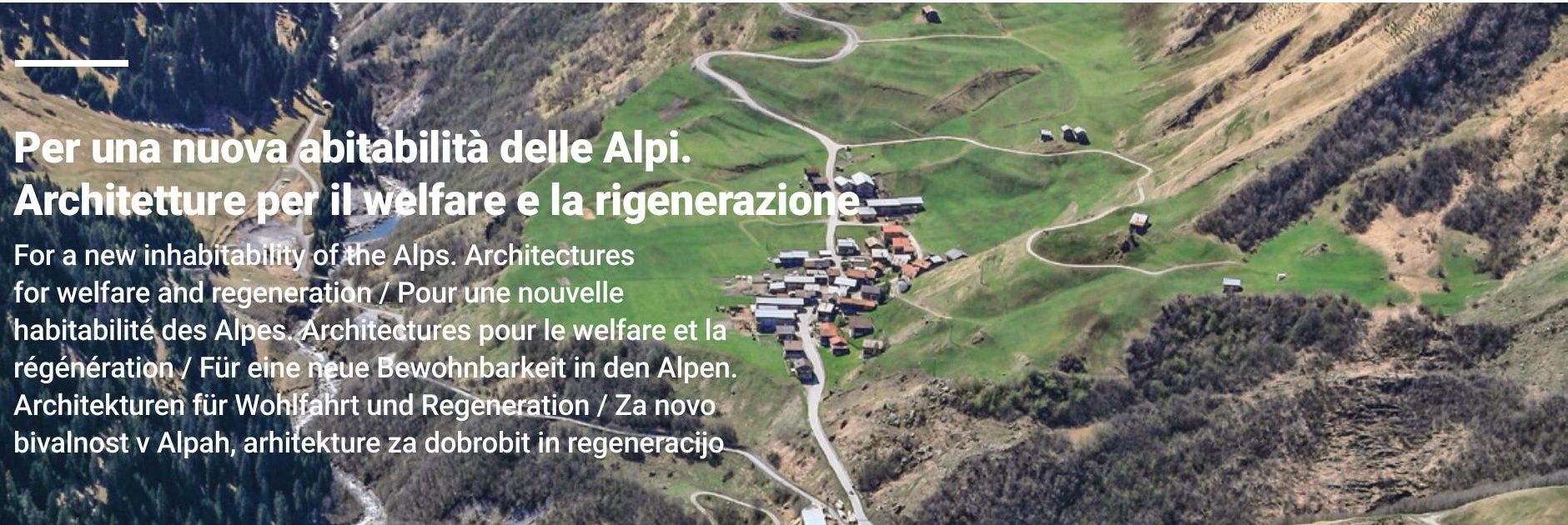
a $x \rightarrow 3$
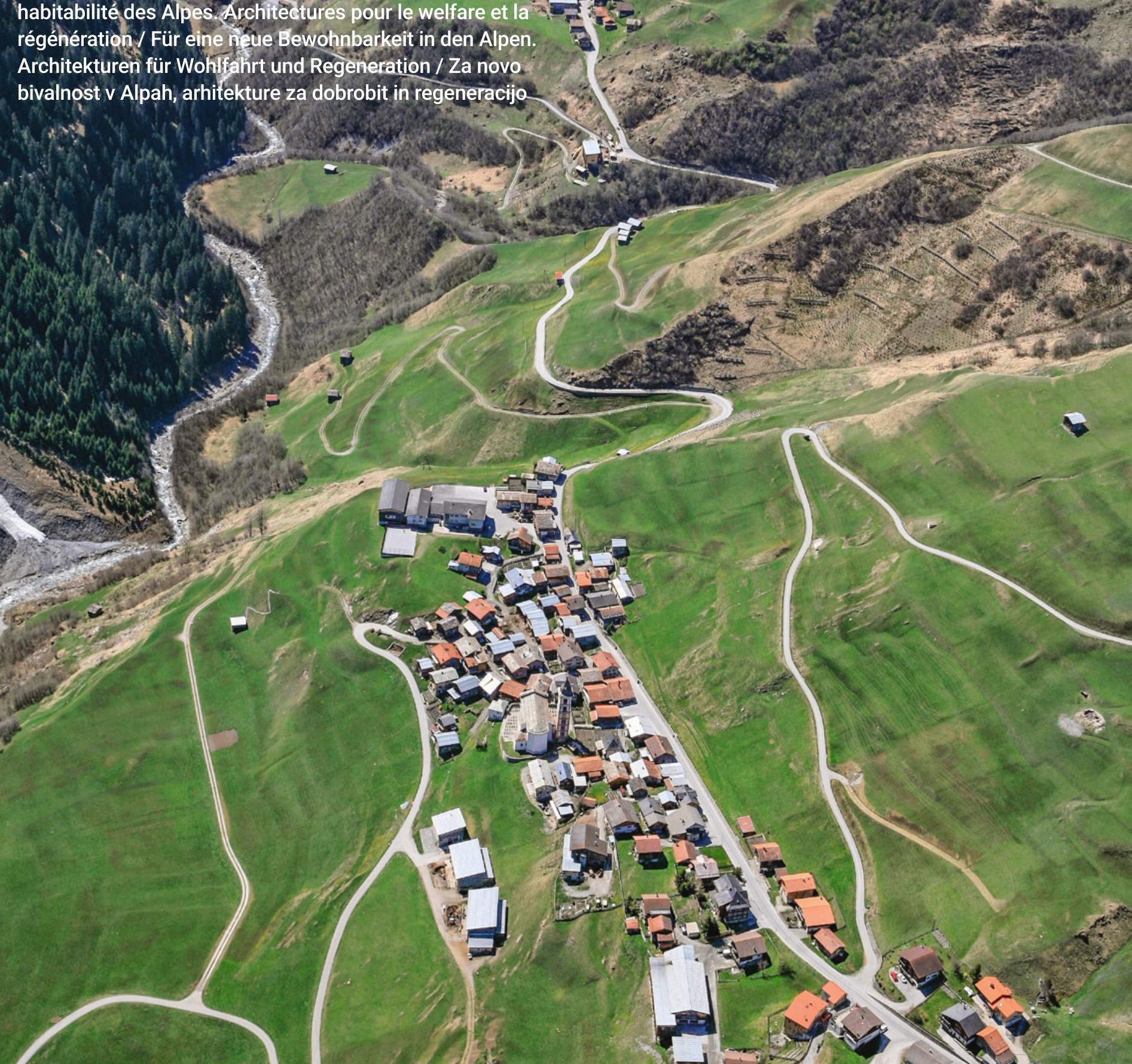


\section{Indice dei contenuti Contents}

\section{Temi}

Sulla centralità di spazio e territorio nel progetto di rigenerazione delle montagne e delle aree interne / On the centrality of space and territory in the project of regeneration of mountains and internal areas Antonio De Rossi, Laura Mascino

I servizi nelle Alpi italiane: quali e dove? Idee per uno scenario post-pandemico / Facilities in the Italian Alps: which ones and where? Ideas for a post-pandemic scenario

Giuseppe Dematteis

Manifesto di Camaldoli per una nuova centralità della montagna

Alla ricerca della distanza perduta. Rigenerare luoghi, persone e immaginari del riabitare alpino / In search of the lost distance. Regenerating places, people and images related to Alpine reinhabitation

Filippo Barbera, Andrea Membretti

Futuro e rigenerazione

Marco Bussone

\section{Esperienze}

Dorferneuerung zwischen Erhalten und Gestalten / 
Valades ousitanes, architettura e rigenerazione / Valades ousitanes,

Ostana e Topolò: hardware, software e welfare nelle comunità di "ritorno" / Ostana and Topolò: hardware, software and welfare in "return" communities

Margherita Valcanover

"Senza mostrare i muscoli": i progetti di rigenerazione di Tao+C e AZL nel solco della "prosperosa società" della Cina contemporanea / "Without flexing one's muscles": the regeneration projects of Tao+C and AZL in the wake of the "prosperous society" of contemporary China Edoardo Bruno, Dalila Tondo

L'archipel Butor. Une régénération, par la culture, d'un village soumis à la métropolisation genevoise / The Butor archipelago. A regeneration, through culture, of a village subject to the metropolisation of Geneva Arnaud Dutheil

Pratiche e progettualità di rigenerazione e welfare: il "Premio triennale Giulio Andreolli - Fare paesaggio" / Actions and projects of regeneration and welfare: the "Premio triennale Giulio Andreolli - Fare paesaggio" Giorgio Tecilla

Architetture e strategie per il welfare. Il caso di Brunico in Val Pusteria / Welfare architectures and strategies. The Bruneck case in Val Pusteria

Eleonora Gabbarini

Arhitektura oživlja / Architecture revives Kristina Dešman, Maja Ivanič

Si Crans-Montana meurt. Soigner le corps malade d'une station / If Crans-Montana dies. Taking care of the ailing body of a tourist resort Patrick Giromini 


\title{
Architetture e strategie per il welfare. Il caso di Brunico in Val Pusteria
}

\author{
Welfare architectures and strategies. \\ The Bruneck case in Val Pusteria
}

\begin{abstract}
In both the South Tyrolean and Alpine areas, the case of Brunico is emblematic with regards to welfare strategies. Since the seventies and up to today, far-sighted planning policies have decisively contributed to their development. The physical and spatial translations of welfare policies and strategies are the several architectural and territorial actions that contribute to providing Brunico with an offer of facilities worthy of a large urban center.

The welfare theme seems to go across all sectors of society. What is particularly surprising is not only the peculiar executive and formal quality of the various infrastructures and facilities available (and planned) in the municipal area, but also, and above all, their concentration within such a limited perimeter.

Careful and minute planning, together with personalized actions on the territory and the adoption of a competition system for the assignment of the projects, all linked by a substantial communion of intent between citizens, the administration and designers, can lead even a mountain city to become competitive in terms of facilities for the community, and welfare strategies and infrastructures.

A possible point of reference for those Alpine realities that want to look to the future with an eye to regeneration and modernity.
\end{abstract}

\section{Eleonora Gabbarini}

Architect and PhD student in "Architecture. History and Project" at Politecnico di Torino, she is undertaking research in the architectural culture in South Tyrol after the 1970s. Passionate about mountain themes, she is also a member of the IAM research center («Istituto di Architettura Montana») and of the editorial staff of the related scientific journal ArchAlp.
Keywords

Architecture, contemporary, landscape, welfare, planning, Bruneck, Val Pusteria. 
Brunico è il nucleo abitato maggiore, nonché capoluogo storico, culturale ed economico del comprensorio della Val Pusteria; dagli anni Settanta del secolo scorso e fino a oggi, a ciò hanno contribuito in maniera decisiva lungimiranti politiche pianificatorie che, dotando il territorio comunale di servizi collettivi, lo hanno configurato di fatto come una valida alternativa al capoluogo di provincia.

Tali politiche si inseriscono nella più generale strategia elaborata nel documento programmatico Alto Adige 'o1 (1973) che, a partire dalla seconda autonomia (1972), vede l'articolazione dell'intero territorio provinciale organizzarsi per centri intermedi di vallata, con l'obiettivo, tra gli altri, "del razionale ed efficiente assetto del territorio provinciale e di un elevato livello di dotazioni sociali e di servizi nelle varie aree della provincia" (Diamantini, 1996). Gli effetti sul territorio di questi atteggiamenti si possono pertanto considerare un catalizzatore della tendenza demografica crescente registrata negli ultimi vent'anni, con una popolazione che è passata dai 13.618 abitanti del 2001 ai 16.716 del 2018 (dati ASTAT).

Secondo Adriano Oggiano, direttore dell'Ufficio Pianificazione Comunale della Provincia Autonoma di Bolzano (intervistato lo scorso marzo), la città di Brunico fa parte di un fertile contesto in cui sono gli abitanti stessi ad interessarsi della cosa pubblica, riconoscendone la ricaduta nel quotidiano di ciascuno; ciò avviene presumibilmente per una consolidata tradizione culturale, improntata ad una forte coscienza civica. Sia in ambito sudtirolese che alpino, il caso di Brunico risulta infatti emblematico per quanto riguarda le strategie di welfare.

Tra i fattori determinanti nel favorire il dispiegarsi di tali processi, va considerato il duraturo e granitico consenso riconosciuto all'amministrazione di

In apertura

II Centro

d'Arrampicata

Brunico/

Kletterzentrum Bruneck,

Stifter+Bachmann Architekten, 2016.

Tutte le fotografie e le elaborazioni grafiche sono di

Eleonora Gabbarini. marca SVP - Südtiroler Volkspartei, ininterrottamente alla guida del comune da decenni.

La traduzione fisica e spaziale delle politiche e strategie per il welfare sono le numerose azioni architettoniche e territoriali che contribuiscono a dotare Brunico di un'offerta di servizi degna di un grande centro; gli attuali standard di elevata qualità diffusa tra gli interventi sembrano potersi ascrivere ad una visione di città tarata sul lungo termine.
Il piano urbanistico della città, "direttamente influente sulle condizioni di vita dei cittadini”" (Linee programmatiche del Sindaco 2014-2020) è stato recentemente rielaborato dall'attuale giunta Griessmair con una procedura iniziata nel maggio 2017 e conclusa nel mese di agosto 2019.

Sempre tra le strategie pianificatorie, il Programma triennale delle opere pubbliche vigente annovera finalità, costi e modalità di realizzazione delle opere architettoniche e infrastrutturali sparse sul territorio, coerentemente con l'ancora precedente Piano Guida/ Leitbild Brunico 2020 (1998), predisposto per mettere a punto, insieme alla cittadinanza, gli obiettivi di sviluppo della città.

Tali documenti strategici sembrano indicare la perseverante volontà di formulare una sorta di disegno d'insieme del futuro di Brunico, elaborato dall'amministrazione comunale rivolgendosi ai cittadini stessi.

Insieme alle strategie ufficiali, pare emergere una sostanziale comunione di intenti che spesso si viene a verificare tra la committenza pubblica e i progettisti locali, motivati dai numerosi bandi di concorso.

Committenza e amministrazione (spesso coincidenti), progettisti e cittadini sembrano concordare dunque su un dato fondamentale di interesse comune: una cultura del costruire qualitativa ed evoluta costituisce un valore sì economico, ma soprattutto sociale, in quanto contribuisce in maniera evidente al benessere della cittadinanza.

Con 1' $82,47 \%$ della popolazione di lingua tedesca (ASTAT, Censimento della popolazione 2011), Brunico presenta storicamente un forte legame con il Tirolo dell'est. Tra i progetti di coordinamento territoriale con il Nord-Est, significativo è l'Interreg-Rat Dolomiti Live, dal 2007 impegnato insieme ai propri partner nell'integrazione dei territori delle regioni transfrontaliere del Bellunese, della Val Pusteria e dell'Osttirol.

A livello comprensoriale, fino agli anni Settanta l'economia è prevalentemente legata all'agricoltura, attività tradizionalmente appannaggio del gruppo linguistico tedesco. Da allora si assiste ad un progressivo sviluppo dell'industria manifatturiera, che si evolve dalle piccole realtà locali della lavorazione 
artigianale ad aziende affermate sul mercato europeo, principalmente attive nel settore delle costruzioni in legno e della meccanica.

Ad esempio, la futura sede di Brunico del Noi Techpark, cluster dell'innovazione e della ricerca, è dedicata al settore automotive, specializzazione sudtirolese particolarmente attiva in Pusteria. La realizzazione del progetto vincitore, a firma dello studio Kerschbaumer Pichler \& Partner di Bressanone, è prevista entro il 2021 sull'area attualmente occupata dalla stazione degli autobus.

Settore economico trainante per la Val Pusteria è sicuramente il turismo, con un'intensità doppia rispetto all'intero territorio provinciale (ASTAT, 2019); di conseguenza, le sue ricadute territoriali e architettoniche sul comprensorio sono particolarmente significative.

Il tema welfare sembra dunque attraversare trasversalmente tutti i settori della società. Ciò che risulta particolarmente sorprendente non è soltanto la particolare qualità esecutiva e formale delle diverse infrastrutture e strutture presenti (e previste) nel territorio comunale, ma anche e soprattutto la loro concentrazione all'interno di un perimetro così limitato.

Fig. 1 Tra gli interventi di infrastrutturazione, già vent'anni fa, nel Piano Guida Brunico 2020 si auspicava l'incentivazione della mobilità dolce e, arrivati alla data di verifica, si può oggi constatare come i $16 \mathrm{~km}$ di piste ciclabili, tratto comunale del più ampio per- corso che collega Bressanone e Lienz, servano sia la popolazione residente sia quella temporanea. Lungo questo asse, sono posizionate stazioni ferroviarie intermedie, attrezzate per consentire l'interscambio tra biciclette e treno.

Lo sviluppo futuro del sistema ferroviario locale è concepito in modo da disincentivare il trasporto privato, garantendo la fermata in molti dei centri sul territorio, con l'obiettivo della drastica riduzione dei tempi di percorrenza. Contestualmente, il sistema "Alto Adige Pass" organizza in modo integrato i trasporti pubblici ferroviari e stradali con il cadenzamento delle corse e l'unificazione delle tariffe. La ferrovia collega poi le stazioni a valle degli impianti sciistici che arrivano al Plan de Corones e consente l'accesso ad importanti servizi alla cittadinanza come l'ospedale.

Da tempo è inoltre prevista la realizzazione del "Centro della mobilità". Peters+Keller Architekten risultano vincitori, nel 2002, del concorso bandito dal Comune con l'obiettivo di riqualificare gli spazi dell'area ferroviaria, elemento di forte divisione del tessuto urbano, trasformandoli in un grande polo multifunzionale integrato.

Questo hub della mobilità, concepito con l'intento di valorizzare il principale punto di interconnessione della Pusteria per il traffico ferroviario e su autobus, non è stato ancora realizzato. Tra le probabili cause di ritardo, i fenomeni di dissesto idrogeologico occorsi in Val Pusteria a fine 2019.

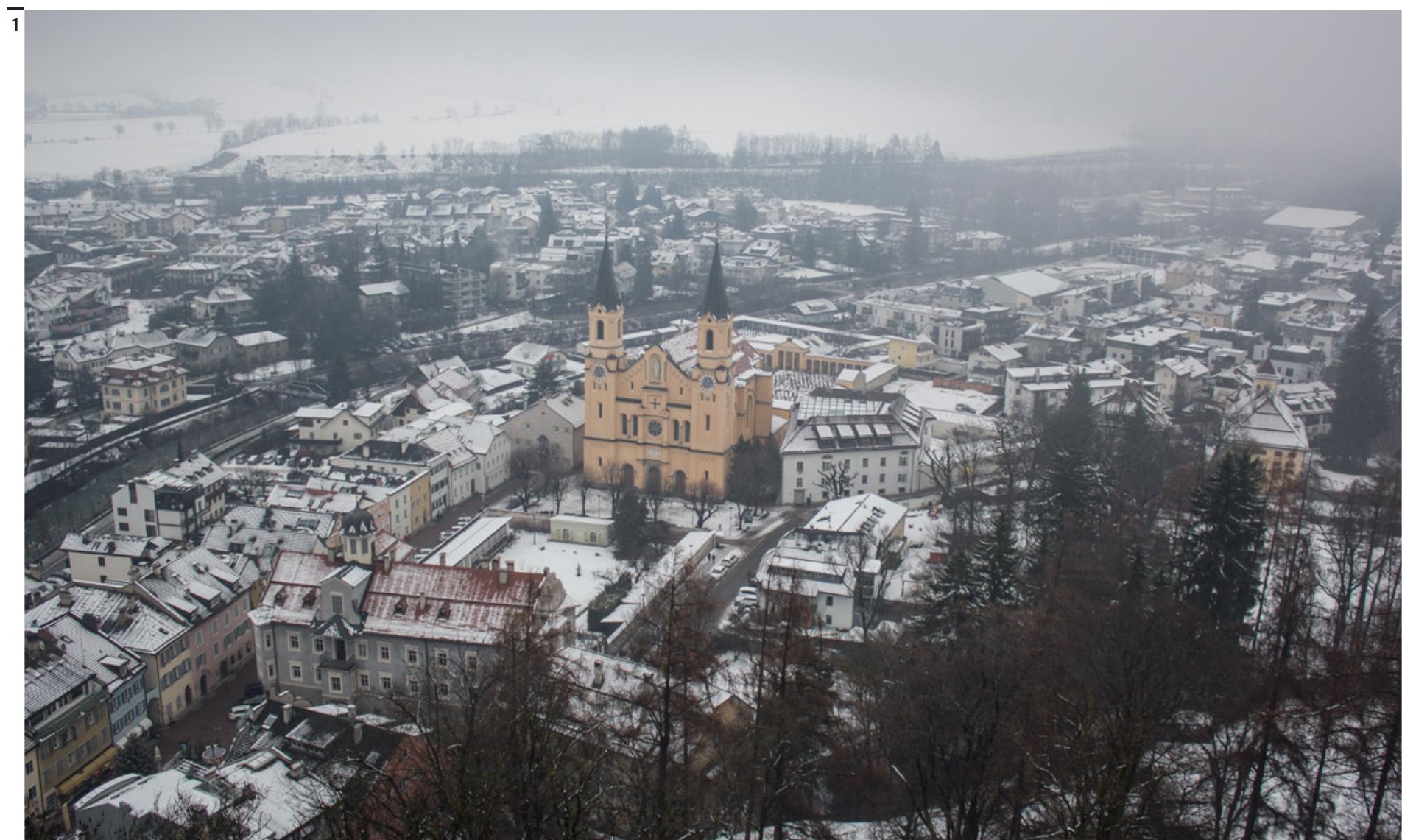


Fig. 2

Brunico e le sue frazioni, planimetria di inquadramento.

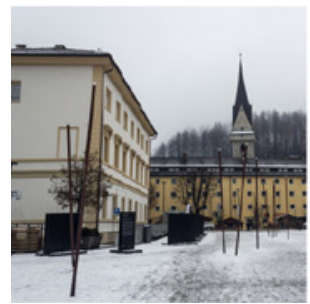

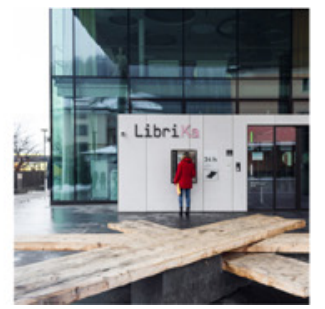

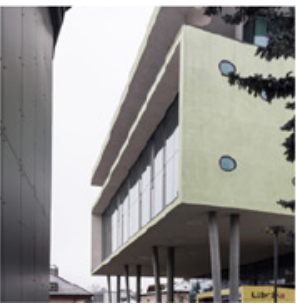

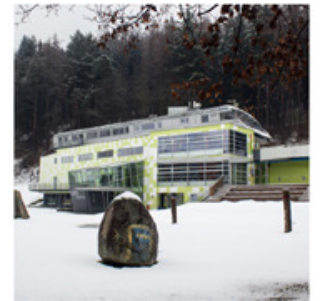

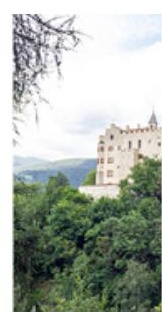

Una seconda categoria di interventi comprende le architetture inserite nel tessuto costruito consolidato di Brunico.

Nel cuore di Brunico città, è situato il nuovo Municipio, costruito insieme alla piazza antistante nel 2005 dagli architetti Abram\&Schnabl. Congiuntamente all'opera d'arte urbana dal titolo "Segni poetici per Brunico", questi interventi concretizzano una suggestiva commistione di arte, spazio urbano e architettura, configurandosi come un legante tra il centro storico e la città nuova.

Già all'interno del documento Alto Adige 2000 e del LEROP - Piano provinciale di sviluppo e coordinamento territoriale del 1995, è previsto uno sviluppo delle dotazioni territoriali policentrico e periferico, dove Brunico va a costituirsi come uno dei poli di riferimento. Di tali dotazioni fanno parte anche le strutture dedicate all'istruzione, con un'offerta didattica che va dalle scuole per l'infanzia fino alla formazione universitaria.

In pieno centro storico, il volume dalle forme semplici e nette della sede locale della Libera Università di Bolzano (le altre due sono a Bressanone e Bolzano) è stato ultimato nel 2007, a conferma dell'importante ruolo dell'edilizia scolastica nello scenario cittadino. A ulteriore riprova della rilevanza di questo settore nell'economia locale, il campus di Brunico ospita il corso di laurea in "Management del Turismo, dello Sport e degli Eventi”.

Rimanendo nel centro storico, l'ampliamento vetrato del liceo delle Orsoline è stato ultimato nello stesso anno su progetto dell'architetto Rudolf Perktold. Tale progetto costituisce un modello di integrazione funzionale nonché di valorizzazione del contesto stradale, per una complessiva riconfigurazione delle aree pubbliche immediatamente adiacenti al lotto scolastico.

Alle pendici della collina dominata dal Castello di Brunico, sorge la nuova scuola di musica. Essa costituisce un centro di importanza sovra-locale per lo studio di questa materia; l'afflusso alle sue attività è tale che, nel 2012, la proprietà comunale indice un concorso per la riqualificazione della preesi- 


\section{$\overline{3}$}

Fig. 3

Brunico città,

planimetria di inquadramento.

Fig. 4

Architetture del welfare a Brunico.
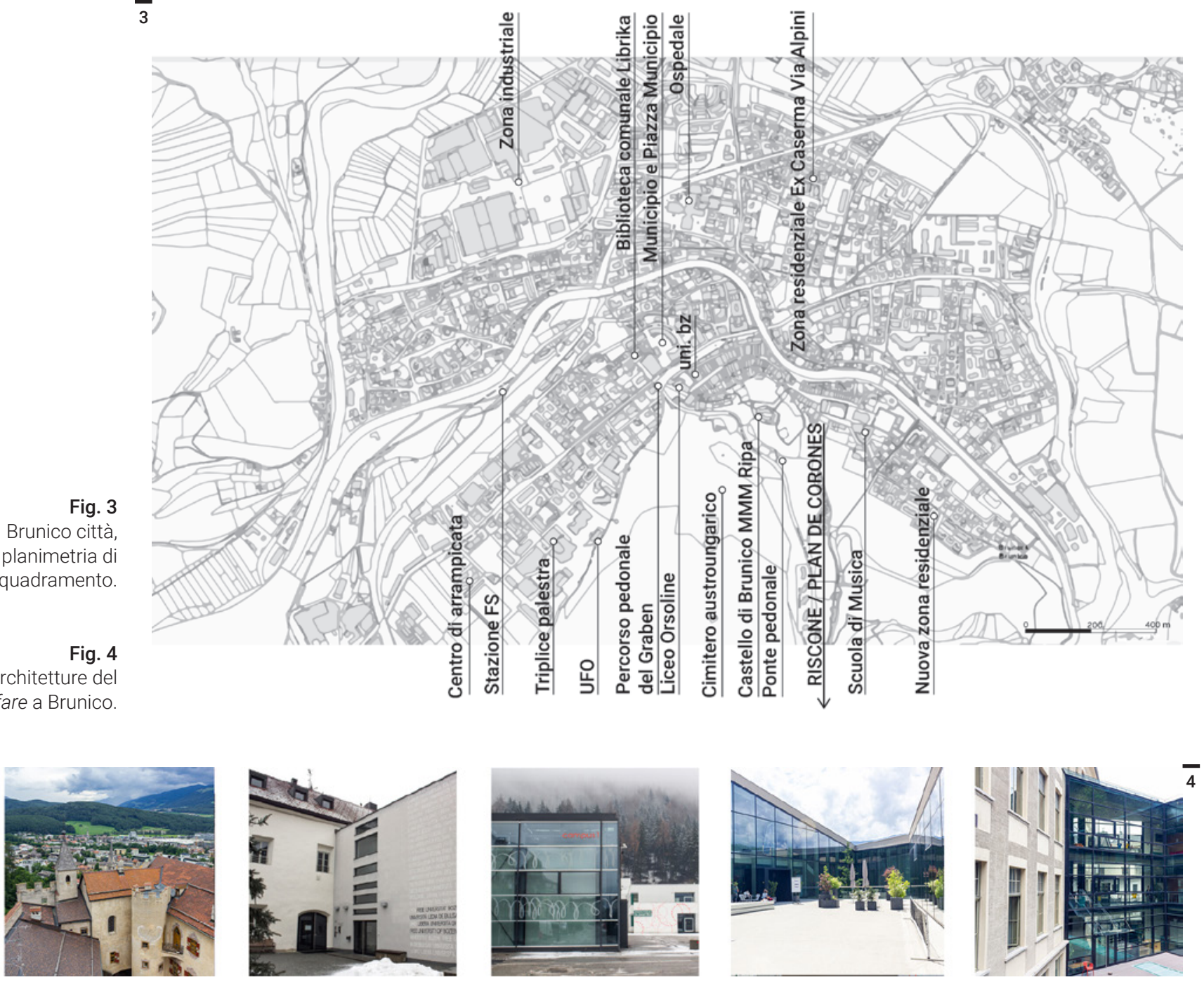

stenza e per un ampliamento. Il progetto vincitore, dello studio italo-spagnolo Barozzi-Veiga, con la valorizzazione degli spazi aperti e il ripensamento del rapporto dentro-fuori, rende il rinnovato complesso particolarmente adatto ad ospitare eventi culturali, mostre e concerti.

Spostandosi più verso la periferia meridionale, si incontra il notevole e diversificato polo scolastico della città.

Oltre al centro culturale giovanile "UFO" ideato nel 1998 da Aichner\&Seidl, al suo interno trovano posto la scuola media con la mensa e la triplice palestra progettata nel 2009 da Konrad Rieper, l'istituto tecnico commerciale, il liceo alberghiero; il tutto viene integrato dalla recente riqualificazione degli spazi aperti messa a punto nel 2012 da Stifter\&Bachmann Architekten.

In particolare, la Dreifachturnhalle (triplice palestra), risultato anch'essa di un concorso, nasce sull'impronta di una delle tre palestre preesistenti, ormai inadatte alle esigenze di versatilità oggi richieste ad un edificio sportivo. Essa è concepita non solo per la fruizione scolastica, ma per un uso aperto all'intera cittadinanza, per lo svolgimento di eventi e manifestazioni.

La valorizzazione complessiva del distretto scolastico coinvolge anche gli spazi esterni, recentemente ripensati da Stifter+Bachmann con aree verdi, alberature e spazi per la sosta e lo svago all'aperto articolati tra le forme curvilinee della nuova pavimentazione.

$\mathrm{Al}$ polo scolastico si accosta una nutrita rosa di spazi per lo sport, tra i quali emerge la nuova palestra di arrampicata. Il Kletterzentrum Bruneck/Centro d'Arrampicata Brunico, inaugurato nel 2016 su progetto di Stifter+Bachmann, è il risultato di un altro concorso pubblico, bandito dalla Provincia per dotare la città di una nuova struttura adeguata al crescente numero di appassionati a questo sport; una necessità espressa anche dal locale club alpino (Alpenverein Südtirol Brunico), con il fine di ospitare i propri corsi e attività. 
Spostandosi verso sud, si incontra la frazione di Riscone, con la quale il conglomerato urbano di Brunico lambisce a sud le pendici del Plan de Corones/ Kronplatz, uno dei principali comprensori sciistici dell'intero arco alpino. Oltre alle decine di migliaia di sciatori che ogni anno frequentano questo vero e proprio parco giochi montano, in tutte le stagioni molti turisti si recano sull'ampia e pianeggiante cima per visitarne le recenti realizzazioni museali. Il discusso progetto di Zaha Hadid per il Messner Mountain Museum "Corones", fiore all'occhiello della rete di musei ideata dal noto alpinista, vede la luce nel 2015. A pochi passi dal MMM, a ridosso della stazione della cabinovia, si colloca il nuovo museo della fotografia di montagna "Lumen", progettato da Gerhard Mahlknecht, Stefano D'Elia, EM2 Architekten \& Giò Forma, Milano (2018) che rivitalizza l'ex edificio funiviario in disuso.

Un'ultima categoria di interventi per il welfare è infine quella delle operazioni "minori" sul territorio, che TB n. 44 definisce i "minimalia".

Nel corso degli ultimi vent'anni, sono molteplici gli interventi di riqualificazione territoriale che hanno contribuito alla valorizzazione tanto della periferia

Fig. 5 urbana quanto del centro storico. Ad esempio, l'aIl Messner Mountain Museum Corones a Kronplatz/Plan de Corones, Zaha Hadid

Fig. 6

Il museo della fotografia di montagna "Lumen", EM2 Architekten, 2018. rea antistante il municipio; la zona situata alla destra orografica della Rienza, compresa tra il ponte di Piazza Cappuccini e il ponte Walde; le zone residenziali, ad est della nuova scuola di musica e in sostituzione della caserma di Via Alpini; infine, il percorso pedonale del Graben, che dal ponte di Piazza Cappuccini conduce al Parco Tschurtschenthaler. Rilevante è anche il ponte pedonale che collega l'area del Castello (convertito nel 2011 da EM2 Architekten nella sede del MMM-Messner Mountain
Museum Ripa) al cimitero militare austro-ungarico posto sul versante boscoso opposto del Kühbergl/ Monte Spalliera.

$\mathrm{O}$ ancora, la già citata area di Riscone, direttamente connessa con il centro storico, sede della stazione di partenza della cabinovia di Plan de Corones e di altri impianti di risalita, del relativo centro servizi, nonché di rilevanti attrezzature sportive che rendono la località in tutte le stagioni dell'anno.

Questi interventi sembrano disegnare un telaio di infrastrutturazione spaziale in cui la sommatoria delle parti va a formare un insieme compatto e solido difficilmente riscontrabile in altre località alpine. Complessivamente, si può dunque ritenere che un'attenta e minuta pianificazione, congiuntamente ad azioni puntuali sul territorio e all'adozione della pratica concorsuale per l'assegnazione degli incarichi, il tutto legato da una sostanziale comunione d'intenti tra la cittadinanza, l'amministrazione e i progettisti, possa condurre anche una località montana a diventare competitiva in termini di servizi alla collettività, di strategie e strutture per il welfare. Queste le ragioni dell'emblematicità di Brunico, forse unica città alpina in contesto italiano dagli spiccati caratteri urbani sviluppati coerentemente con le istanze sociali e le ormai imprescindibili istanze di salvaguardia ambientale.

Un possibile punto di riferimento per quelle realtà alpine che vogliono guardare al futuro in un'ottica di rigenerazione e di contemporaneità.

Si ringrazia l'arch. Adriano Oggiano, direttore dell'Ufficio Pianificazione comunale della Provincia Autonoma di Bolzano-Alto Adige, per le informazioni e il supporto, indispensabili per la realizzazione di questo testo.

\section{Bibliografia} Abram Zeno, Schröder Nina, Tschurtschenthaler Christian (2005), «Rathausplatz Bruneck», in Turris Babel 66 -
Plätze / Piazze, aprile 2005.
Attardo Paola (2008), «Municipio di Brunico», in Turris Babel 75 - Rathäuser / Municipi, febbraio 2008.
D’Affronto Andrea (a cura di) (2002), «Centro mobilità di Brunico. Concorso per la riqualificazione della zona
ferroviaria», in Turris Babel 55 - Öffeltliche Bauten / Edilizia pubblica, maggio 2002 .
Dellago Stephan (2011), «Wettbewerb Kletterhalle Bruneck», in Turris Babel 85 - Wettbewerbe / Concorsi, aprile 2011.
Diamantini Corrado (a cura di) (1996), Gli ambienti insediativi del Trentino e dell'Alto Adige, Università di Trento, Di-
partimento di Ingegneria Civile e Ambientale, Trento.
Fondazione Architettura Alto Adige (a cura di) (2013), Guida d'architettura dell'Alto Adige, Edition Raetia, Bolzano.
Gabbarini Eleonora (2020), Intervista ad Adriano Oggiano, 12 marzo 2020.
Istituto Provinciale di Statistica (2019), Alto Adige in cifre, Provincia Autonoma di Bolzano.
Kunst Meran (2018), New Architecture in South Tyrol 2012/2018 / Neue Architektur in Sudtirol 2012/2018 / Archi-
tetture Recenti in Alto Adige 2012/2018, Park Books, Zürich.
Schwärzer Johann (2001), «Wettbewerb Dreifachturnhalle Bruneck», in Turris Babel 54 - Drauf und dran, novem-
bre 2001.

Winterle Alberto (2018), «Anonimi silenzi. La scuola di musica di Brunico», in Turrisbabel 110 - Architettura sonora, luglio 2018

https://www.comune.brunico.bz.it/it/

https://astat.provincia.bz.it/it/default.asp

https://www.sta.bz.it/it/stazioni-treni/progetti-in-programma/

http://www.provincia.bz.it/costruire-abitare/edilizia-pubblica/opere-ultimate/

https://www.suedtirol.info/it/esperienze/

https://www.unibz.it

https://www.keep.eu/project/5228/interreg-rat-dolomiti-live 
$\overline{5}$

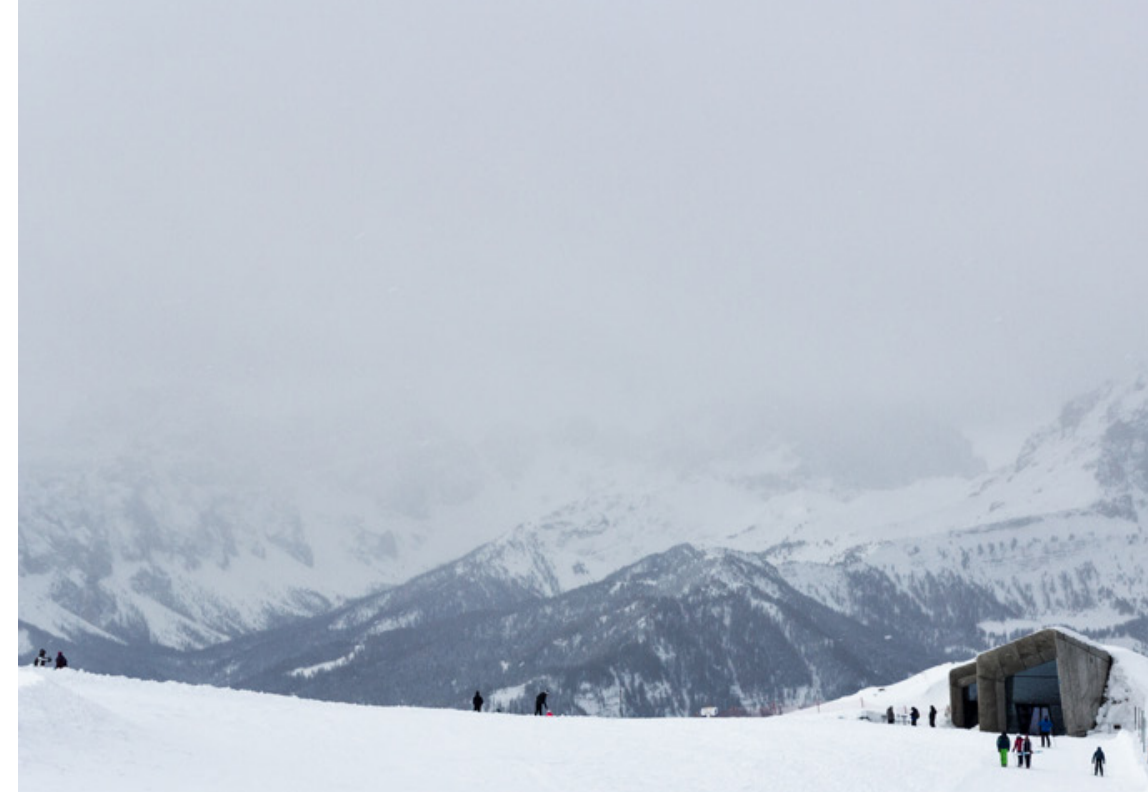

R

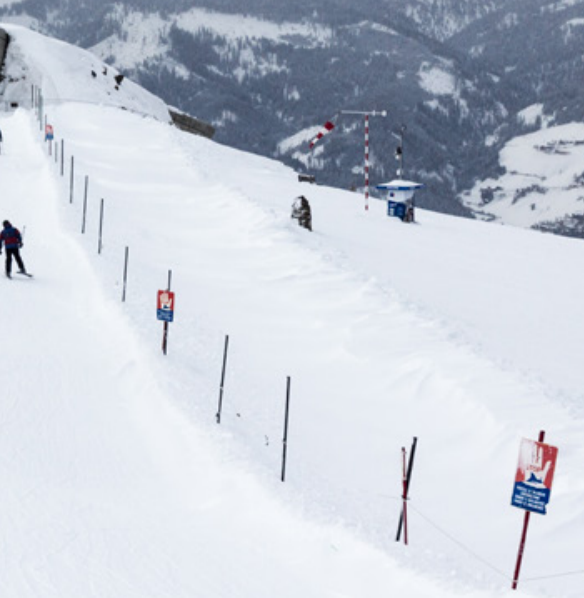

$\overline{6}$

1

1 这

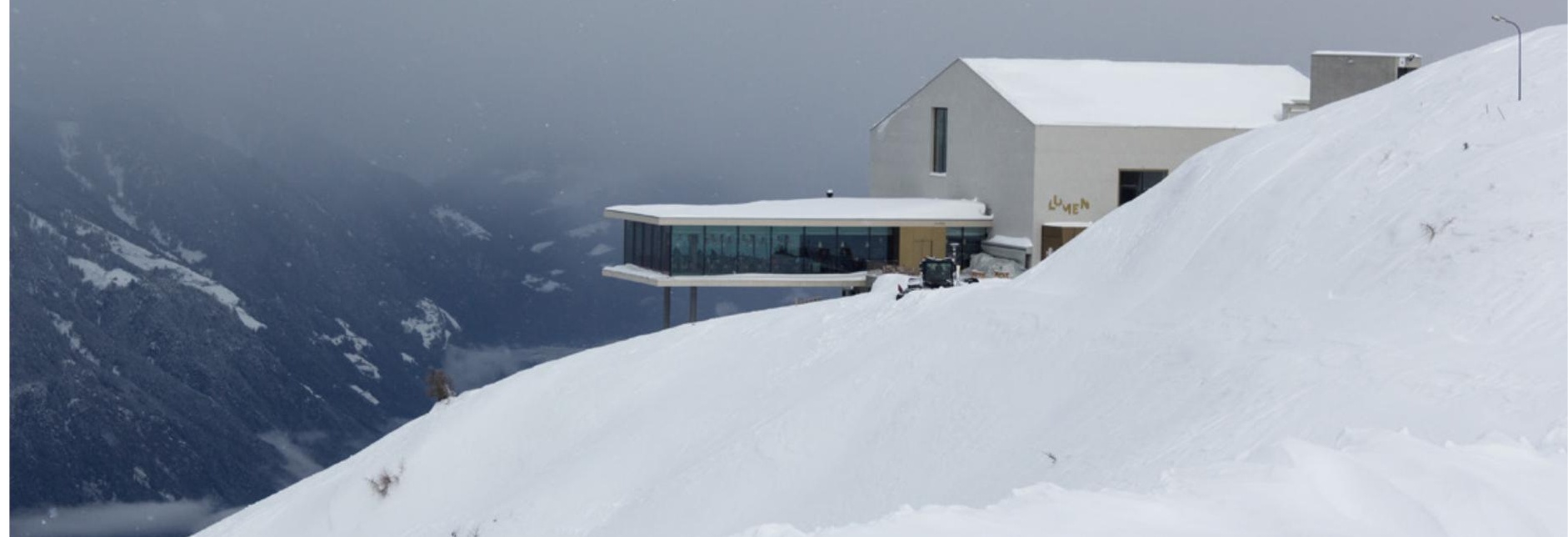

